\title{
La atención a pacientes con trastornos mentales en las prisiones
}

\section{Introducción}

Muchas de las apreciaciones y opiniones que exponemos proceden de la experiencia española, tanto de los servicios públicos de salud mental en la red sanitaria general, como de los servicios de sanidad penitenciaria. No es dificil,sin embargo, intentar desde ahí una aproximación a la perspectiva europea, ya que la mayor parte de los problemas, y de las posibles soluciones, son muy parecidos de un país a otro.

Es cierto que, tal y como se recoge en las Recomendaciones $\mathrm{N}^{\circ} \mathrm{R}(98), 7 \mathrm{del}$ Comité de Ministros del Consejo de Europa, en los últimos años, coincidiendo con el aumento de la población penitenciaria, se ha observado un sensible incremento en el número de personas que padecen algún tipo de trastorno mental. Esto no significa que sea debido a un efecto específico de la prisión sobre las personas internas sino más bien, de una parte, a la desaparición de los manicomios como espacio que durante dos siglos ha albergado a una buena cantidad de personas socialmente problemáticas, $y$ por otro lado, al considerable aumento de la población general que carece de recursos de todo tipo o está simplemente marginada, entre la que hay que contar con un buen número de personas con trastornos mentales, en especial psicosis crónicas, que de una manera u otra acaban con facilidad en la prisión. Sin contar, claro está, con los trastornos mentales provocados por el consumo de sustancias tóxicas o por enfermedades como el SIDA.

A ello hay que sumar esa serie de problemas descritos en el documento de La Haya ${ }^{4}$, como riesgos de suicidio, problemas por adaptación al medio penitenciario, los derivados de la frecuente masificación de centros -incapaces de garantizar el principio básico de privacidad e intimidad del interno y, especialmente, su seguridad-, de sus malas condiciones materiales, higiénicas, etc,.., de la escasez de personal o su deficitaria formación, de la ausencia de información adecuada sobre los diferentes aspectos de la vida en el centro estrictamente reglamentados, de la arbitrariedad con que se hagan cumplir tales reglamentos, el aislamiento prolongado o indefinido, los malos tratos físicos o psicológicos, el posible incumplimiento de las normas relativas a los derechos fundamentales de las personas internadas, los traslados arbitrarios de cárcel, el internamiento en prisiones alejadas del medio familiar, etc.

\section{Fundamentos y contexto legislativo}

$1^{\circ}$ La Constitución Española (1978), al igual que otras constituciones, 
reconoce explícitamente el derecho a la salud de todos los ciudadanos

\section{$2^{\circ}$ La Ley General de Sanidad} (1986) reconoce el derecho a recibir atención a los problemas de la saludmetal sin ninguna discriminación respecto a otros problemas de salud. El Sistema Nacional de Salud atenderá a los pacientes mentales en la misma red asistencial que el resto de los pacientes ( en España no hay una legislación sanitaria específica diferenciada para la asistencia psiquiátrica)

$3^{\circ}$ El Código Penal español, tras la reforma de 1995, estipula que cuando una persona considerada ininputable por causa de enfermedad mental ,haya cometido un delito penal, el Tribunal tiene la posibilidad de someterle a un tratamiento ambulatorio, o podrá imponerle internamiento psiquiátrico ( involuntario) por un periodo que en ningún caso será superior al de la pena que hubiera podido corresponderle.

\section{La realidad asistenciales en las prisio- nes}

\subsection{Aspectos generales}

1) En España hay una población en prisiones en torno a 40.000; es decir , cerca de uno por mil de la población general.

2) Casi el 5\% de esta población sufre trastornos mentales psicóticos ( 1920 pacientes, según datos de la Dirección general de Instituciones Penitenciarias..)

3) Además, 420 pacientes están en uno de los dos hospitales psiquiátricos penitenciarios existentes en el Estado, y otra cantidad de pacientes no precisada, en unidades de psiquiatría de la red sanitaria general. En ambos casos se trata de sujetos considerados culpables de delitos graves, $y$ reconocidos como inimputables por el Tribunal. En estos casos el juez ha indicado el internamiento como medida de seguridad cuya finalidad es el tratamiento y la rehabilitación ( el que el paciente sea enviado a una unidad de hospitalización psiquiátrica general o a un hospital psiquiátrico penitenciario, depende del juez).

Como quedó dicho anteriormente, la estancia en el hospital ( ya sea psiquiátrico pentenciario, ya psiquiátrico general), en ningún caso podrá ser superior a la que el sujeto hubiera debido permanecer en prisión, de haber sido considerado imputable.

\subsection{Premisas para una correcta atención}

Cualquier plan para la atención de las personas privadas de libertad con trastornos mentales debe pasar por asumir las tres premisas siguientes:

A. La persona privada de libertad mantiene su derecho a la salud, como el resto de la población general.

B. El enfermo mental no debe sufrir ninguna discriminación con respecto al resto de los enfermos (lo que no siempre es asumido por los responsables de las prisiones o los médicos de las mismas; $y$ no es infrecuente que se nieguen a tratar enfermos mentales y tiendan a trasladarlos rápidamente a un hospital psiquiátrico, sobre todo si hay uno penitenciario).

C. El Sistema Nacional de Salud de cada país debe implicarse plenamente en la atención a los problemas sanitarios de los presos, y 
concretamente la atención a sus problemas de salud mental, de la misma manera que lo hace con el resto de su población (en caso contrario, los enfermos presos y el personal sanitario de las prisiones se encuentran doblemente aislados y por tanto con menos posibilidades de afrontar con éxito los problemas que se plantean $y$, sobre todo, de encontrar soluciones a largo plazo.

Aunque parezca obvio, ello supone que toda la normativa que existe en leyes nacionales sanitarias o penitenciarias y reglamentos específicos, incluyendo las leyes penales, deben hacer alusión expresa a estos principios y ser revisadas no solo para facilitar su cumplimiento, sino también para suprimir cualquier obstáculo que suponga una discriminación con respecto a la población general o a los enfermos de las restantes patologías. Hay leyes penales que consideran aún, que durante el tiempo en que un interno sufra una enfermedad psiquiátrica, queda suspendida su pena, que tendrá que terminar de cumplir una vez recuperada "la razón", lo que, entre otras cosas -caso de una demencia SIDA- puede suponer la condena indefinida.

De acuerdo con los principios citados, la atención psiquiátrica en las prisiones estaría basada en los siguientes puntos:

1. La base del sistema sanitario de la prisión debe constituirla un equipo de atención primaria, formado por médicos, enfermeros y personal auxiliar cualificado, quedando la psiquiatría en un segundo nivel.
2. Como señala el punto 2 del Anexo a las Recomendaciones $n^{\circ} R(98) 7$, dichos equipos deben contar con suficiente personal para asegurar un mínimo de atención ambulatoria y de urgencias a todo el personal internado que lo necesite y para poder realizar planes de detección precoz de los problemas psiquiátricos o preventivos.

3. Estos equipos de atención primaria deben ser los responsables de la detección, tratamiento inicial y si es posible resolución de la mayor parte de los problemas de salud mental que sucedan en la prisión. La experiencia española demuestra que cuando los internos acceden directamente a un especialista en psiquiatría, no solo demandan muchos más fármacos tranquilizantes o estimulantes sino que existe una mayor tendencia a psiquiatrizar -también por parte de los responsables de la prisión- la mayor parte de los conflictos habituales en estas instituciones totales.

4. De acuerdo también con la experiencia española, consideramos de la máxima importancia implicar a los equipos de los centros de salud mental en la comunidad (pertenecientes al Sistema Nacional de Salud), para que sean ello quienes presten el correspondiente apoyo psiquiátrico y psicológico a dichos equipos de atención primaria. En términos generales se puede afirmar que en este caso existe una mejor calidad asistencial que cuando es la propia prisión quien contrata al psi- 
INFORMES

quiatra, que en ningún caso estará en condiciones de realizar las prestaciones del equipo comunitario. Además tiene la ventaja de que los equipos comunitarios pueden hacer después un seguimiento y control de los casos que lo requieran, cuando se concede la libertad al preso, libertad que muchas veces se ordena por el juez de la noche a la mañana, sea cual sea el estado clínico del paciente (es decir, es importante hacer ver a los responsables comunitarios que los problemas sanitarios del personal recluso, antes o después se trasladan a la comunidad).Sin embargo, los psiquiatras de centros penitenciarios $y$ pocas veces contacta con los servicios de la comunidad.

5. Es misión fundamental de los equipos de salud mental (o de los responsables sanitarios de la comunidad, si estos equipos no existen) la formación continuada de los equipos de a.p. de la prisión, fundamental para que estos puedan realizar con mayor solvencia y confianza el tratamiento oportuno de los problemas de salud mental. Formación que reclaman normalmente y que pocas veces se les da.

6. Es indispensable la existencia de camas de psiquiatría en las enfermerías de las prisiones, para lo que es fundamental que todo nuevo establecimiento cuente con una moderna enfermería y que se mejoren al máximo las de 10 s establecimientos antiguos. Sin ello es muy difícil garantizar una correcta asistencia a los casos agudos que casi siempre son tratados en celdas de aislamiento, que reúnen muy malas condiciones.

7. En los casos más graves, es recomendable que el enfermo sea trasladado a la Unidad de Psiquiatría del Hospital más próximo, de la red sanitaria general.

8. Un problema de difícil solución es el de los psicóticos crónicos (y los retrasos mentales más o menos severos). Se trata de personas, a menudo abandonadas por sus familias, víctimas ya en la calle de ciertos clanes, pero que carecen de recursos personales para sobrevivir en el duro ambiente de la prisión. Su incapacidad para cumplir las estrictas reglas de la cárcel no solo les hace víctimas frecuentes de castigos, sino que en la mayoría de los casos son los que nunca pueden acceder a los beneficios del llamado tratamiento penitenciario, que en realidad consiste en un código de buena conducta que cuando se cumple, reporta, especialmente sustanciales reducciones de condena.

Para este colectivo es preciso constituir equipos de rehabilitación que desde la comunidad y con la colaboración de los funcionarios ( a quienes convendría preparar e incentivar para el desarrollo de estas tareas), pueden llevar a cabo programas de rehabilitación encaminados precisamente a mejorar la adaptación de estos enfermos a la vida penitenciaria, su participación en todo tipo de actividades que ofrezca la prisión, laborales, educativas, 
deportivas, etc. y responsabilizarlos a su vez para su reinserción posterior en la comunidad. Pero hay que ser conscientes de que la rigidez de las estructuras penitenciarias, la desconfianza con la que los funcionarios viven la entrada de personas ajenas a la prisión y a menudo también la resistencia de los jueces a otorgar beneficios, precisamente a enfermos mentales por el dichoso estigma, hacen muy difícil la constitución y funcionamiento de tales equipos.

Alguna experiencia concreta, como la que se está llevando a cabo con pacientes de la cárcel de Navalcarnero, en Madrid, avalan este enfoque.

9. Por eso es también primordial la formación de los funcionarios en un doble sentido: porque ellos están necesitados de programas especiales de promoción de salud y prevención por el tipo de trabajo que realizan (especialmente los que han de tratar con casos violentos), pero también de cara a los enfermos mentales para informarles debidamente y ayudar a luchar contra el estigma de estos enfermos, que pocas veces son realmente conflictivos (exceptuando los serios trastornos de la personalidad), pero son mal vistos por dificultades de adaptación a ciertas rutinas, o por no colaborar en algunas tareas cotidianas (limpiar su celda, cumplir horarios o participar en actividades).

10. Tanto para el abordaje de los problemas de salud mental dentro de la prisión como para los derivados de su puesta en libertad, es fundamental la cooperación de los servicios sociales de dentro y de fuera de la prisión.

11. Un problema especial es el de la existencia o no de hospitales psiquiátricos penitenciarios teniendo en cuenta que existe un grupo de población que habiendo cometido un delito grave, es privada de libertad por decisión judicial, aunque no se le considere responsable. En España, por ejemplo, se llevó a cabo el desmantelamiento del viejo hospital penitenciario psiquiátrico, dirigido por funcionarios no médicos, con escasa dotación sanitaria y cuyas condiciones infrahumanas fueron condenadas públicamente por la AEN y el Defensor del Pueblo y se suprimió también un centro para psicópatas, que había sido fuente de continuos problemas, incluyendo graves motines incapaces de controlar por los funcionarios, y lógicos por concentrar a los reclusos más conflictivos. Alternativamente se construyeron dos nuevos establecimientos penitenciarios psiquiátricos, dotados de personal especializado (psiquiatras, psicólogos, trabajadores sociales, rehabilitadores, enfermeros y auxiliares).

Se modificó también el Código Penal, en los aspectos antes comentados, suprimiendo los artículos que hacían indefinido el internamiento psiquiátrico, evitando lo que tan a menudo estaba sucediendo en la práctica: que la mayoría de los que ingresaban en una de estos establecimientos vivía en él hasta su muerte. 
Cuando se produjo la revisión de todos estos internos, la mayoría llevaba internado muchísimos años por pequeños delitos, casi siempre robos-. La aplicación del actual Código Penal español, que data de 1995, ha contribuido a disminuir notablemente la población psiquiátrica ingresada por este motivo y a evitar situaciones poco acordes con los derechos humanos de muchos enfermos. Al mismo tiempo se ha reglamentado que en los establecimientos psiquiátricos penitenciarios solo se podrá ingresar por decisión de un juez penal, suprimiendo la posibilidad de que pueda ser trasladado un recluso de una prisión cualquiera por decisión de su director o del cuerpo médico.

\section{Consideraciones finales y conclusiones}

4.1 Hemos podido comprobar que la frecuencia de conductas delictivas por personas que padecen trastornos mentales mantiene una estrecha relación con las condiciones sociales ( las situaciones difíciles tales como el paro, la carencia de hogar y vivienda con el consiguiente vagabundeo en la calle, la emigración - legal e ilegal- , los treaumas biográficos, el haber sido víctima de abusos e incluso de tortura... la pobreza, además del abuso y/o dependencia del alcohol u otras drogas ). Todas ellas constituyen situaciones de riesgo tanto para sufrir enfermedad mental como para incurrir en conductas delictivas... Por tanto:

$1^{a}$ Conclusión. Tener en cuenta que las medidas preventivas básicas son de naturaleza social y política; son por tanto una responsabilidad socio-política, antes que responsabilidad social o sanitaria. Aún así, corresponde también a los servicios de salud (general y penitenciaria) reclamar la adopción de esas medidas.

4.2 Por otra parte, los problemas de la estructura social, tales como la debilidad de los lazos sociales y de valores como la solidaridad, desplazados por el individualismo y la competitividad, constituyen sin duda factores que contribuyen a mantener la marginación de grandes grupos de población, incluyendo muchos pacientes mentales. Es especialmente llamativo que aún en lugares donde existen, accesibles a la población, servicios sociales y de salud mental, las personas más pobres y marginadas, que son quienes previsiblemente tienen necesidades más importantes, no llegan a conectar muchas veces con estos servicios, por lo que:

$2^{\mathrm{a}}$ Conclusión. Hay que reforzar la estructura y la organización de los servicios sociales, de los servicios de atención primaria, y de los servicios de salud mental comunitaria para disminuir las barreras ( culturales, administrativas..) y facilitar el acceso a los servicios, especialmente a la población en riesgo de exclusión o claramente excluida. A este respecto, es imprescindible reconocer los derechos de ciudadanía a todos los que en un momento dado se encuentran en territorio europeo. 
INFORMES

4.3 Comprobamos también el problema tan frecuente de los pacientes que tras su estancia en prisión, al ser puestos en libertad, se interrumpe le tratamiento, no establecen contacto con el servicio de salud mental de referencia, y a menudo no volverán a estar en tratamiento hasta que vuelven a prisión, tras la recaida - la repetición de las conductas delictivas y quizás en recaída clínica... Por lo que:

$3^{\text {a }}$ Conclusión. Se debe establecer una estrecha colaboración entre los servicios médicos de las cárceles y los equipos de salud metal comunitaria, al menos antes de que el pacientepreso sea puesto en libertad, con objeto de preparar la recepción comunitaria y facilitar el acceso a los programas, en igualdad de condiciones que para el resto de pacientes mentales crónicos de su lugar de residencia.

4.4 Se deben prestar una particular atención a las condiciones del medio carcelario, con el fin de disminuir los riesgos de vivir en una institución cerrada/total, y reforzar al máximo las posibilidades de realización personal aunque sea dentro de un medio tan restrictivo . Así, por ejemplo : es imprescindible atajar la superpoblación de la que hemos hablado ayer o, de acuerdo con el documento de conseso propuesto por la OMS, en su reunión de la Haya (noviembre de 1998),

$4^{\text {a }}$ Conclusión, adoptar medidas de promoción de la salud mental, y tratamiento psiquiátrico adecuado, tales como:

- Adoptar medidas terapéuticas dirigi- das a promover la resistencia emocional del paciente

- Reforzar sus habilidades sociales

- Facilitar la comunicación emocional Hoy sabemos que ciertos métodos de psicoterapia individual, así como otros de tipo grupal o familiar, son absolutamente necesarios para hacer posible la recuperación de la psicosis. El enfermo mental en la prisión debe tener acceso a estos tratamientos; no debería haber ninguna razón para que ésto no fuera sí.

4.5 En el interior del medio carcelario, el paciente psicótico no está en una situación simétrica respecto a los demás; está en una situación de precariedad de recursos interpersonales, quedando a menudo expuesto al riesgo de ser objeto de abuso por parte de los demás, por lo que es necesario que alguien se responsabilice de la tarea de "tutela" o de defensa de sus intereses en la vida cotidiana de la prisión. Hay que formar e incentivar a quienes puedan desempeñar esta importante misión ( 5 a Conclusión a)

Otra dimensión del problema es que muy a menudo los pacientes mentales no accdeden a los beneficios penitenciarios, como ya hemos dicho antes. Al respecto, proponemos que se considere la posiblidad de obtener beneficios penitenciarios vinculados al desarrollo de los programas de rehabilitación psicosocial, incorporando los posibles beneficios al proceso terapéutico ( de acuerdo a la situación particular de cada paciente) ( $5^{\text {a con- }}$ clusión b) 


\subsection{6 $^{\text {a }}$ Conclusión: Consideramos muy} necesario que se lleven a la práctica las citadas Recomendaciones $\mathbf{R}(98)$ del Comité de Ministros del Consejo de Europa; muy especialmente en lo que se refiere a los siguientes aspectos:

- Independencia de los facultativos para tomar sus decisiones

- Prohibición de prácticas denigrantes(camisa de fuerza, por ejemplo)

- Puesta en práctica de reglas estrictas en lo que se refiere a las medidas de inmovilización ( que deben quedar restringidas a aquellos casos de urgencia en los que, provisionalmente, no pueda hacerse otra cosa)

- En cuanto a los derechos del enfermo, facilitar una buena información previamente a que pueda aceptar el tratamiento, evitar el abuso de medicación, etc

- Prestar una adecuada atención a las necesidades clínicas: en la evaluación inicial, en los momentos de crisis, y más específicamente en la atencion a los pacientes crónicos

- «Los detenidos que sufran alteraciones mentales graves deberían ser ubicados y cuidados en un servicio hospitalario dotado de equipamiento adecuado y de personal cualificado. La decisión de admitir un detenido en un hospital público debería ser tomada por un médico psiquiatra, y con la autorización de las autoridades competentes" (R 55)

- «En los casos en que el aislamiento de los enfermos mentales no pueda ser evitado, éste debería ser reducido a una duración mínima y reemplazado lo antes posible por una vigilancia de enfer- mería permanente y personal".( R56)

- «Los riesgos de suicidio deberán ser valorados de forma permanente por el personal médico y penitenciario. Durante los periodos críticos, según los casos, se deberían adoptar medidas de contención física concebidas para evitar que que los enfermos-detenidos se puedan hacer daño a sí mismos, vigilancia estrecha y permanente y apoyo relacional" ( R58)

4.7. Respecto a la organización de servicios.

En la situación europea actual, en la que la mayoría de los paises disponen de una organización dividida entre la red sanitaria general y la penitenciaria - con algunas excepciones , como es el caso de Noruega; y de otra forma Francia, Italia, y parcialmente, España- con servicios funcionalmente diferenciados y más o menos alejados.

$7^{\mathrm{a}}$ Conclusión : Consideramos indespensable ponerse a trabajar en común, los servicios de la red sanitaria general y los de la red penitenciaria - al menos en lo que respecta a la atención a pacientes que padecen trastornos mentales- a través de programas de coordinación y de cooperación con, al menos, los siguientes objetivos:

$\left.1^{\circ}\right)$ Reforzar la independencia de la actuación clínica y de la perspectiva de los profesionales de la salud allí cuando se de una confrontación con la perspectiva de las instituciones cuando tienden a priorizar la defensa de la seguridad.

$2^{\circ}$ ) Preparar el seguimiento, tras la puesta en libertad, como ya hemos señalado antes 
$3^{\circ}$ ) Compartir los programas de formación continuada, y propicar que se comparta una cultura pareja sobre la intervención rofesional... es necesario avanzar sobre la combinación, el mestizaje de la cultura penitenciaria con la asistencial-rehabilitadora

$\left.4^{\circ}\right)$ La colaboración se debe ampliar hacia otros trabajadres de las prisiones no profesionales de la salud. Es necesario proporcionarles formación continuada y ayuda psicológica también contnuada para facilitar el afrontamiento del estrés y la relación con los prisioneros en las mejores condiciones posibles.

Finalmente, proponemos reconsiderar seriamente la propuesta expuesta por varios ponentes de que la salud penitenciaria sea un programa de la salud general que dependa sustancialmente del Ministerio de la Salud
Aún otra esperanza : el movimiento asociativo de pacientes, de ex-pacientes, de familiares, en varios paises de Europa están interesándose por la situación y el porvenir de los pacientes mentales que están en prisión. Nosotros, los profesionales, al menos los de la Asociación Española de Neuropsiqiatría y los de Salud Mental Europa, nos sentimos implicados y comprometidos en común también en este proyecto.... "Si la idea del desmantelamiento de los manicomios surgió de la consideración de su falta de eficacia terapéutica y de levantar el ocultamiento de verdaderos depósitos de miseria humana, nosotros debemos hacer ahora un esfuerzo para que los centros penitenciarios no se conviertan en los nuevos "depósitos de locos"s

${ }^{1}$ Psiquiatra. Vicepresidente de Salud Mental Europa/Federación Mundial de Salud Mental. Presidente de la Asociación Española de Neuropsiquiatría.

${ }^{2}$ Psiquiatra. Ex-asesor de salud mental en instituciones penitenciarias, Ministerio de Justicia.

${ }^{3}$ Presentado en la conferencia multilateral organizada por el Consejo de Europa - Programa de actividades para el desarrollo y la consolidación de la estabilidad democrática. Estrasburgo. Diciembre 1999.

4 "Declaración de consenso sobre la promoción de la salud mental en las prisiones"( noviembre 1998), promovido por la Oficina Regional Europea de la Organización Mundial de la Salud, aprobado por la asamblea general de Salud Mental Europa/FMSM en Bruselas el 27 de marzo de 1999.

s Comentarios de un alto funcionario de la Dirección General de Asuntos Penitenciarios, según se cita en : "Informe especial: situación de los enfermos mentales en centros penitenciarios andaluces". Defensor del Pueblo Andaluz - Sevilla DPA 1997 ( publicado en el BOPA n 193.Comisión n² 220. Serie A9-12-1998). Disponible en internet: http://defensor@defensor-and.es/i-mental.htp 